\title{
Genotyping-by-sequencing based cytoplasmic markers underpin population structural variation of perennial ryegrass
}

\author{
Mingshu $\mathrm{Cao}^{1 *}$, Marty Faville ${ }^{1}$, Jeanne Jacobs ${ }^{2}$, Marcelo Carena ${ }^{1}$ \\ ${ }^{1}$ AgResearch Grasslands Research Centre, Palmerston North 4442, New Zealand \\ ${ }^{2}$ AgResearch Lincoln Research Centre, Christchurch 8140, New Zealand
}

\section{*Correspondence:}

Mingshu Cao mingshu.cao@agresearch.co.nz

Keywords: Cytoplasmic markers, Chloroplasts, Mitochondria, Genotyping-by-Sequencing, Lolium perenne

\begin{abstract}
Chloroplast and mitochondrial genomes provide unique information in studying plant populations because cytoplasmic genes exhibit a different mode of inheritance and a different rate of gene mutation compared to nuclear genes. Despite this, cytoplasmic genomic contributions to plant population performance are largely unexplored because few methods are available to characterize and evaluate cytoplasmic genome-wide variations. Here we have developed cytoplasmic markers based on genotyping-by-sequencing (GBS), which enable us to characterize thousands of samples, to survey gene variants across cytoplasmic genomes, and to monitor within-population variations of chloroplast or mitochondrial origin. Using these cytoplasmic genome-wide markers we have found that withinpopulation differentiations are evident in ryegrass (Lolium perenne), beyond the explanation of nuclear markers. Moreover, chloroplast and mitochondrial variations exhibit different patterns, with mitochondrial markers more readily reflecting the maternal origins. Application of GBS-based cytoplasmic markers should facilitate quantifying the contribution of cytoplasmic inheritance to plant performance through selective breeding or under natural selection pressure.
\end{abstract}

\section{Introduction}

Plant cytoplasmic genomes of chloroplasts and mitochondria provide unique information for studying population diversity because genes of cytoplasmic origin exhibit a different mode of inheritance and a different rate of gene mutation compared to nuclear genes (Wolfe et al. 1987). Thanks to their relatively small size complete mitochondrial or chloroplast genomes have been sequenced for 3,015 plant species, according to the NCBI organelle genome databases (https://www.ncbi.nlm.nih.gov/genome/organelle/ accessed at April 2019). Organelle genome information has contributed to our understanding of phylogenetic relationships or evolution among many plant species. That information was based on variations estimated from complete chloroplast DNA (cpDNA), mitochondrial DNA (mtDNA), or targeted genes residing in cytoplasmic genomes 
(Hiesel et al. 1994; Dobler et al. 2014; Daniell et al. 2016). At the level of microevolution (i.e. from the perspective of plant improvement), cpDNA and mtDNA sequence-informed genetic markers, such as simple sequence repeat (SSR) markers, have been used to study plant domestication, to validate the parental origin of commercially important varieties, and to characterize genetic variation among germplasms or cultivars (Galtier et al. 2009; Daniell et al. 2016). Nonetheless, all such studies were based on markers derived from a few genes or repeat regions, and on a limited number of individual plants due to the limited capability of genotyping methods.

In breeding for outcrossing plant species, selections are often carried out at the population or family level. Accurately monitoring gene allele frequency allows desirable genotypes to predominate in selected populations, and this calls for efficient genotyping tools that are deployable at a large scale. Genotyping-by-sequencing (GBS) (Elshire et al. 2011), due to its multiplexing and use of restriction enzyme(s) to reduce genome complexity, represents one of the productive and affordable options for characterizing a large number of samples to meet the demand for genetic improvement of populations. We have applied GBS to characterize perennial ryegrass (Lolium perenne L.) populations based on nuclear DNA (nuDNA) derived single nucleotide polymorphism (SNP) markers (Faville et al. 2018). Given a massive amount of sequencing tags sampled from each individual plant we anticipate that a certain number of tags will be derived from cpDNA and mtDNA, thus could be utilized for cytoplasmic SNP discovery. Organellar DNA is expected to be contained in routine whole genomic DNA isolations (Islam et al. 2013), and organelle genomes could even be assembled from whole genome data (Dierckxsens et al. 2017).

In perennial ryegrass the mitochondrial genome (Islam et al. 2013) and chloroplast genome (Diekmann et al. 2009; Hand et al. 2013) have been assembled. In those reports perennial ryegrass chloroplast and mitochondrial genomes were studied in comparison with that from more or less related species, the purposes of which were to gain insights into phylogenetic relationships. More relevant to the context of our investigation here is whether these types of variations can be harnessed at the level of cultivar or germplasm (of the same species). Polymorphism was indeed found among a few individual $L$. perenne plants from the same population based on cpDNA-derived microsatellite markers (Diekmann et al. 2012), or based on SNPs detected from chloroplast sequencing reads (Diekmann et al. 2009). However, no systematic information is available on the extent of cpDNA and mtDNA variation occur within ryegrass cultivars or among cultivars. With its scalability and free of ascertainment bias GBS may provide an opportunity to assess such genetic variation within and among populations.

With an aim to explore and exploit that opportunity we have developed a computational workflow for haploid SNP discovery from mapping existing GBS reads to cytoplasmic reference genomes (cpDNA 
and mtDNA); we quantified the genetic similarity of individual plants and assess genetic polymorphism within ryegrass breeding populations using distance metrics. Potential uses of GBSbased cytoplasmic markers for breeding outcrossing and perennial plant species are discussed.

\section{Results}

\section{Identification and characterization of cytoplasmic markers from ApeKI GBS libraries}

We used GBS data derived from two ryegrass breeding populations 'P96' (112 plants) and 'P127' (120 plants). Four controls samples from the same DNA extraction of a single individual were used to assess the reproducibility of SNP discovery.

A total of 5,115,152 quality tags were generated from the 236 samples. Only 8,807 unique tags were mapped onto the cytoplasmic reference genomes, among which 6,056 and 2,751 tags were mapped to cpDNA and mtDNA, respectively. 1,071 raw haploid SNPs were obtained using samtools and bcftools. Fewer SNPs (197) were detected with cpDNA as the reference than the number of SNPs (874) detected with mtDNA. The two sets of SNPs are hereafter referred to as cpSNPs and mtSNPs.

The control samples, although of the same DNA extraction, were subjected to four independent GBS library preparations and sequenced in different batches (sequencing flowcells and lanes). 95.3\% (1021 out of 1071) SNPs were consistently identified ( $\mathrm{sd}=0, \mathrm{n}=4)$ among the controls, demonstrating the reliability of SNP calling. The 50 inconsistent SNPs appeared to be random in the controls, and its variation was not associated with the low read depth (mean of read depth of the $50 \mathrm{SNPs}=644.7$ ). We thus removed these inconsistent SNPs. Among the retained SNPs, 649 were called with the constant value of 1 across both populations, being 1 means the site genotype has a different nucleotide from the corresponding reference site (see Methods). Although different from the reference the monomorphism among the samples under the study offers no information we therefore removed them from further analysis. As a result, 372 SNPs (100 cpSNPs and $272 \mathrm{mtSNPs})$ were retained for statistical analysis.

Phi correlation coefficient $(\phi)$ was applied to measure genetic relationships of the samples based on the retained SNPs. Multidimensional scaling (MDS) analysis performed on the distance metric (1- $\phi)$ showed somewhat complex relationships between populations and within each population, based on both cpSNPs and mtSNPs (Fig. 1). 


\section{a: based on $100 \mathrm{cpSNPs}$}

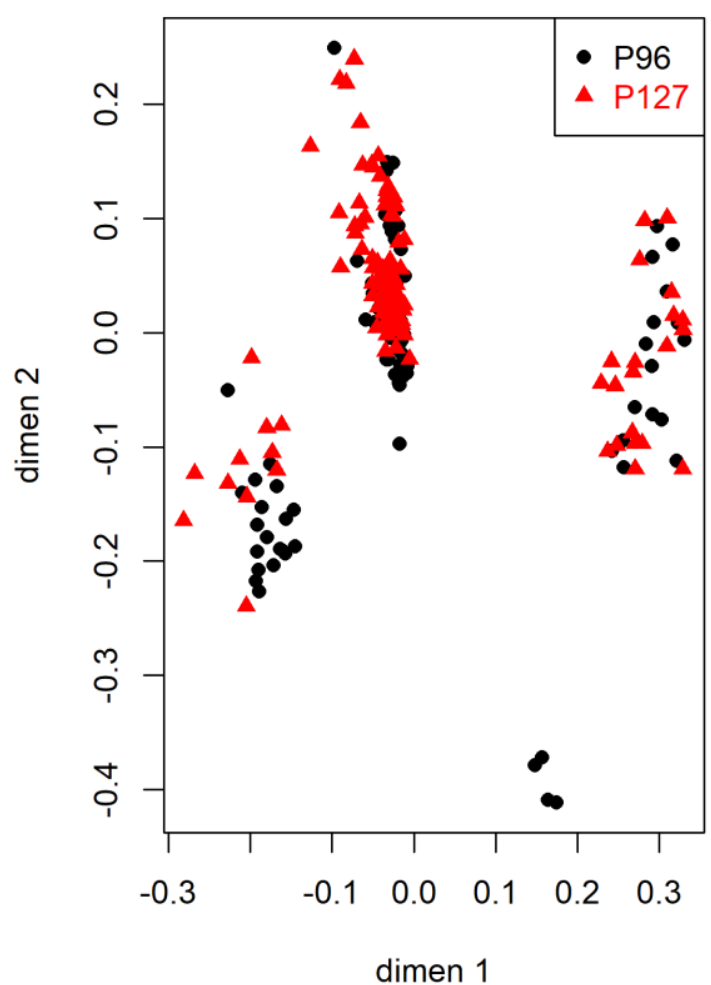

b: based on $272 \mathrm{mtSNPs}$

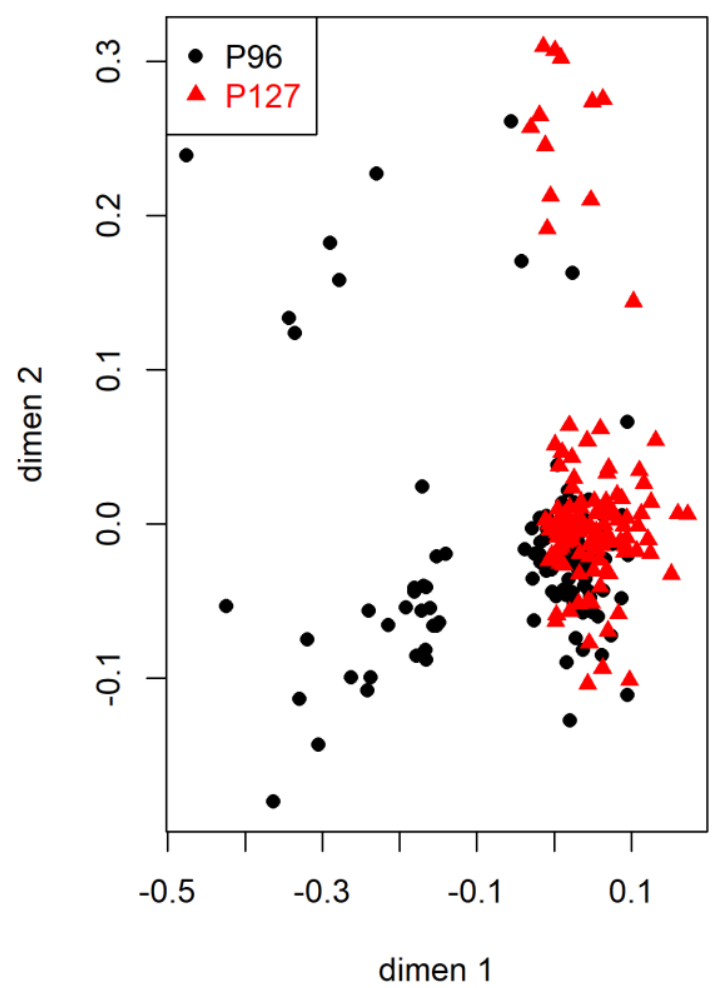

Fig. 1 Multidimensional scaling clustering of samples from perennial ryegrass populations P96 $(\mathrm{n}=112)$ and P127 ( $\mathrm{n}=120)$, based on cpSNPs (a) and mtSNPs (b).

Substructures within both 'P96' and 'P127' populations were clearly revealed based on cpSNPs (Fig. 1a). It was unexpected to see that the sample clustering did not reflect the two populations, with three clusters nonetheless observed only within the respective population (Fig. 1a). Sanity checking via principal component analysis confirmed the substructures were not associated with the GBS libraries, sequencing batches, nor the amount of tags per sample. On the other hand, based on mtSNPs 'P96' largely formed two clusters across the dimension 1, with discernible within-population differentiations (Fig. 1b).

Excluding possible confounding technical factors (such as the library and sequencing batches) the substructure revealed should be attributed to the intrinsic SNP variations. A close inspection of the variation patterns of cpSNPs among the samples confirmed that this was the case. There were 3 clusters of plants within 'P96' (Fig. 1a and Supplementary Fig.1), where, as shown in Fig. 2 (limited view due to the space), plants $35,38,44,48,58,60,63$ belonged to the same class as the same pattern of SNP variation was presented. 


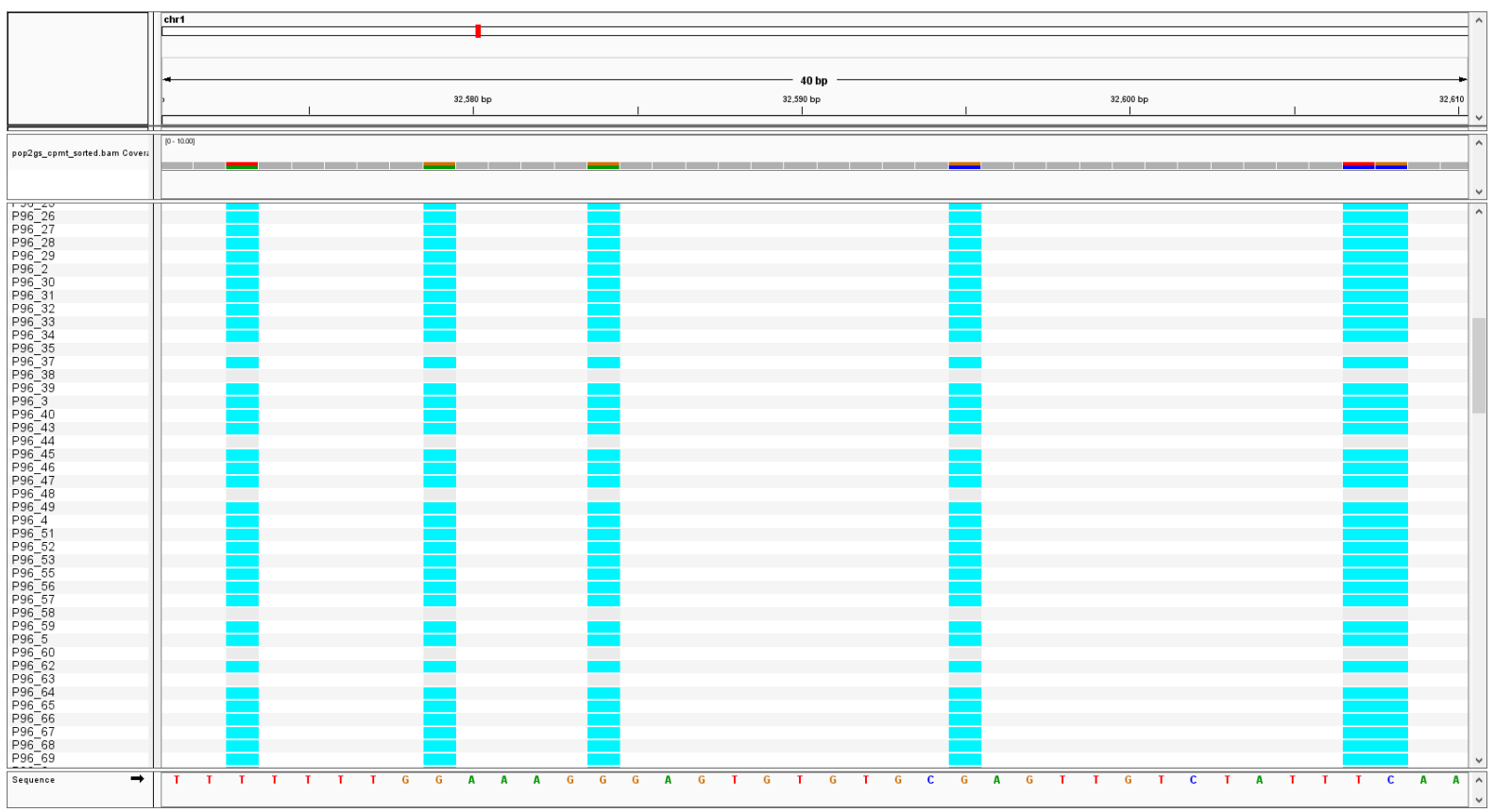

Fig. 2 SNP variation among samples of ryegrass population P96, as shown in a region of cpDNA where six SNPs are called; plants P96-35, 38, 44, 48, 58, 60, 63 exhibit different SNP variations compared to the other plants. For example, P96-35 (gray) are the same as the reference at all six SNP sites, while P96-37 (blue) has different site genotypes from the reference.

With an interest to identify SNPs that are differentially distributed between the two populations we performed statistical testing with the R function "prop.test". There were 99 SNPs identified as significant (Benjamin \& Hochberg adjusted p-value < 0.01), out of which 15 were cpSNPs and 84 mtSNPs. A detailed analysis was conducted as follows regarding the correlation and origin of these significant SNPs.

\section{Correlation and origin of cpSNPs}

Comparing to Hamming distance the phi coefficient has an intuitive interpretation as Pearson's correlation coefficient, in that the coefficient is ranged from -1 (perfect negative association) and 1 (perfect positive association). We performed a hierarchical clustering analysis of the $15 \mathrm{cpSNPs}$ based on phi coefficient (1- $\phi)$, and the result was shown in Fig. 3. Two negatively correlated groups were largely formed for cpSNPs. In general, adjacent cpSNPs (the SNP location is encoded in the ID), such as cp68818 and cp68834, cp48913 and cp48940 were perfectly correlated, a trend often observed for nuclear SNPs (nuSNPs). However, cp77036 and cp77039 were adjacent SNPs but negatively correlated $(\phi=-1)$ across samples of the two populations. This revealed a fact that plants with number genotype 0 (see Methods) at cp77036 must have genotype 1 at cp77039 (Table 1). 


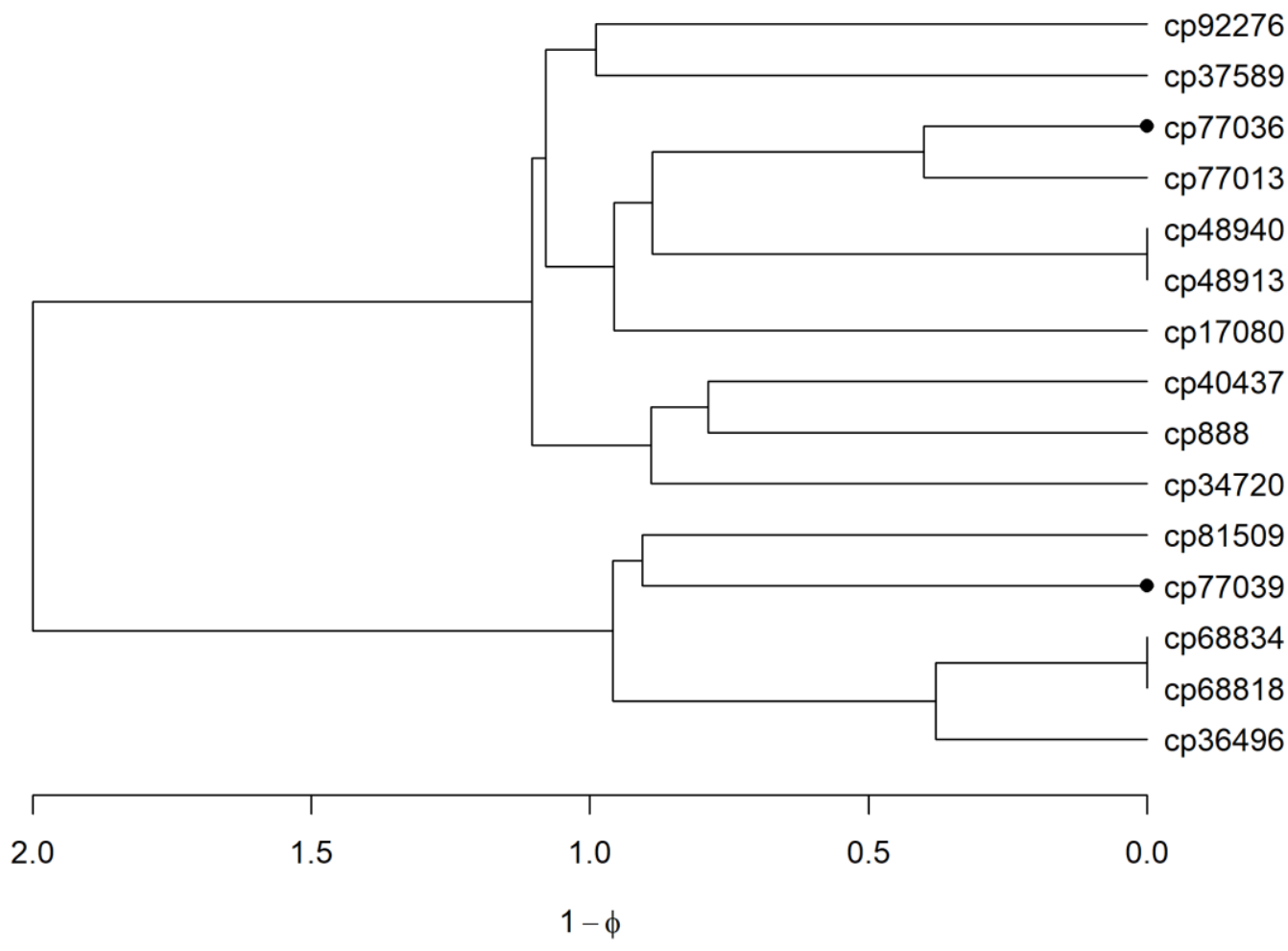

Fig. 3 Hierarchical clustering of 15 cpSNPs based on distance metric (1- $\phi)$. Two SNPs (cp77036 and cp77039) as dotted are adjacent but negatively correlated in contrast with other adjacent SNPs.

Table 1 Population diversity and annotation of cpSNPs. A summary of 15 cpSNPs including column information: $\boldsymbol{i d}$, SNP id with prefix cp denoting chloroplast origin, and integer denoting its physical position in the chloroplast genome; $\boldsymbol{r e f}$, reference site base; $\boldsymbol{a l t}$, detected alternative base. $\boldsymbol{D P}$, read depth; P96div, SNP genotype may be population-dependent, for example, cp34720 has genotype C > $\mathrm{T}$ in $19 \%$ of plants in the population P96, as shown P96div score $=0.19 ; \boldsymbol{P 1 2 7 d i v}$, this SNP cp34720 has only genotype $\mathrm{C}>\mathrm{C}$ in all $\mathrm{P} 127$ plants, with P127div score $=0 . \mathrm{cp} 36496$ is an indel also presenting as population-specific; annotation, gene symbols, with the full name listed in the Abbreviation. 


\begin{tabular}{|c|c|c|c|c|c|c|}
\hline$\underline{i d}$ & $r e f$ & alt & $D P$ & P96div & P127div & annotation \\
\hline $\operatorname{cp} 888$ & $\mathrm{C}$ & $\mathrm{A}$ & 274 & 0.04 & 0.18 & psbA \\
\hline cp17080 & G & $\mathrm{A}$ & 72 & 0.02 & 0.12 & petN \\
\hline cp34720 & $\mathrm{C}$ & $\mathrm{T}$ & 109 & 0.19 & 0 & $\operatorname{atpA}$ \\
\hline cp36496 & CAAAAAAAA & CAAAAAAAAA & 117 & 0.12 & 0 & unknown \\
\hline cp37589 & A & $\mathrm{G}$ & 155 & 0 & 0.09 & psaB \\
\hline cp40437 & A & $\mathrm{T}$ & 485 & 0.02 & 0.22 & psaA \\
\hline cp48913 & $\mathrm{C}$ & $\mathrm{G}$ & 221 & 0.05 & 0.19 & ndhK \\
\hline cp48940 & $\mathrm{G}$ & $\mathrm{A}$ & 221 & 0.05 & 0.19 & ndhK \\
\hline cp68818 & $\mathrm{C}$ & A & 2642 & 0.28 & 0 & psbB \\
\hline cp68834 & $\mathrm{C}$ & $\mathrm{G}$ & 2642 & 0.28 & 0 & psbB \\
\hline cp77013 & $\mathrm{C}$ & $\mathrm{A}$ & 43 & 0 & 0.08 & rp114 \\
\hline cp77036 & $\mathrm{C}$ & $\mathrm{G}$ & 158 & 0.03 & 0.15 & rp114 \\
\hline сp77039 & $\mathrm{G}$ & A & 158 & 0.03 & 0.15 & rp114 \\
\hline cp81509 & $\mathrm{C}$ & $\mathrm{T}$ & 99 & 0.27 & 0.05 & unknown \\
\hline cp92276 & A & $\mathrm{G}$ & 484 & 0.44 & 0.25 & unknown \\
\hline
\end{tabular}

It was our observation that some SNP genotypic variation was population-dependent. For example, cp68818 had genotype C > C, (a letter genotype denoting ref base > site base, see Methods) for all 'P127' plants. However, this SNP showed differentiations in 'P96', with 81 plants genotyped as C > $\mathrm{C}(0)$ and 31 plants genotyped as $\mathrm{C}>\mathrm{A}(1)$.

To quantify such SNP variations within a population we defined SNP diversity score as:

$$
\operatorname{div}=\min (\# 0, \# 1) / \text { population size }
$$

For each population, \#0 refers to the number of plants with genotype 0 and \#1 the number of plants with genotype 1. For example, for cp68834, its div score in 'P96' (P96div) was 0.28 (31/112) while in "P127" the div score $=0 . \mathrm{P} 127 \mathrm{div}=0$ indicated no differentiation within that population. The maximum SNP div score in a population is 0.5 . The div scores for the top 15 cpSNPs were provided in Table 1. Although no difference on average in the diversity score across all 15 cpSNPs (P96div = $0.12, \mathrm{P} 127 \mathrm{div}=0.11)$ there were obvious differences between populations at each individual SNP, which manifested in the population substructures observed in Fig. 1a. Gene psbB (see Abbreviations for the gene symbol description) showed a strong differentiation in 'P96' only, while psbA, ndhk and rpl14 genes were more diverse in 'P127'. An indel (cp36496), located upstream of gene psaB (36513 - 38717), was differentiated in 'P96' only. cp92276 of unannotated origin exhibited a high degree of variation in both populations. 


\section{Correlation and origin of mtSNPs}

Likewise, we conducted the investigations on mtSNP diversity within the populations. The clustering of the 84 significant mtSNPs (Supplementary Fig. 2) presented some redundant information in that the adjacent SNPs were highly, positively correlated. For simplicity and without the loss of information, we merged the highly correlated $(\phi>0.9)$ and adjacent $(<64 \mathrm{bp})$ SNPs for discussion. The remained $57 \mathrm{mtSNPs}$ formed two distinct groups (Fig. 4). One group contains $21 \mathrm{mtSNPs}$ which were characterized as P127-diverse (div scores, mean $=0.11$, sd $=0.08$ ), i.e. homogeneous in 'P96' (mean $=0.05, \mathrm{sd}=0.06$ ). Another group of $36 \mathrm{mtSNPs}$ is a P96-diverse group with P96div (mean $=0.15, \mathrm{sd}$ $=0.096)$ and P127div $($ mean $=0.05, \mathrm{sd}=0.09)$.

Summarized in Table 2 included the diversity score and annotation of the $57 \mathrm{mtSNPs}$. Annotations for the mtSNPs are scarce due to the fact that about $79 \%$ of the ryegrass mitochondrial genome sequences have not been annotated (Islam et al. 2013). In addition to the population-specific mtSNP variations we also made the following observations: (1) For adjacent SNPs, although highly positive correlation was the main trend, the variations in the level and even direction of correlation did occur: from $\phi=-1$ for pairs such as mt42400/42408 and mt366077/366087, to low correlation pairs such as $\mathrm{mt} 379742 / 37974$ ( $\phi=-0.04), \mathrm{mt335734/335736}$ ( $\phi=-0.13)$ and $\mathrm{mt675467/675498}(\phi=0.08) ;(2)$ SNPs derived from rrn18 gene were heterogeneous in 'P96' but remained homogeneous in 'P127', showing population-specific type of variation; in contrast, SNPs from atp4 appeared to be random between the populations; (3) The most variable region in the mitochondrial genome were in the rrn18 gene, within which both $\mathrm{rrn} 5$ and ccmFC are resided; (4) $19 \mathrm{mtSNPs}$ (including adjacent and correlated SNPs) were found from position 580,440 to 676,066 bp, indicating another variable but unannotated region. We performed a RepeatMasker analysis (with Poacea database)(Tarailo-Graovac and Chen 2009; Bao et al. 2015), and found that this region hosts a number of microsatellite sequences and LTR/Gypsy (Supplementary data 2). (5) Last but not least is the low SNP diversity scores (as also relevant for Table 1). It deserves a further investigation on whether the low div scores e.g. $<0.05$ are considered as the noise level. 


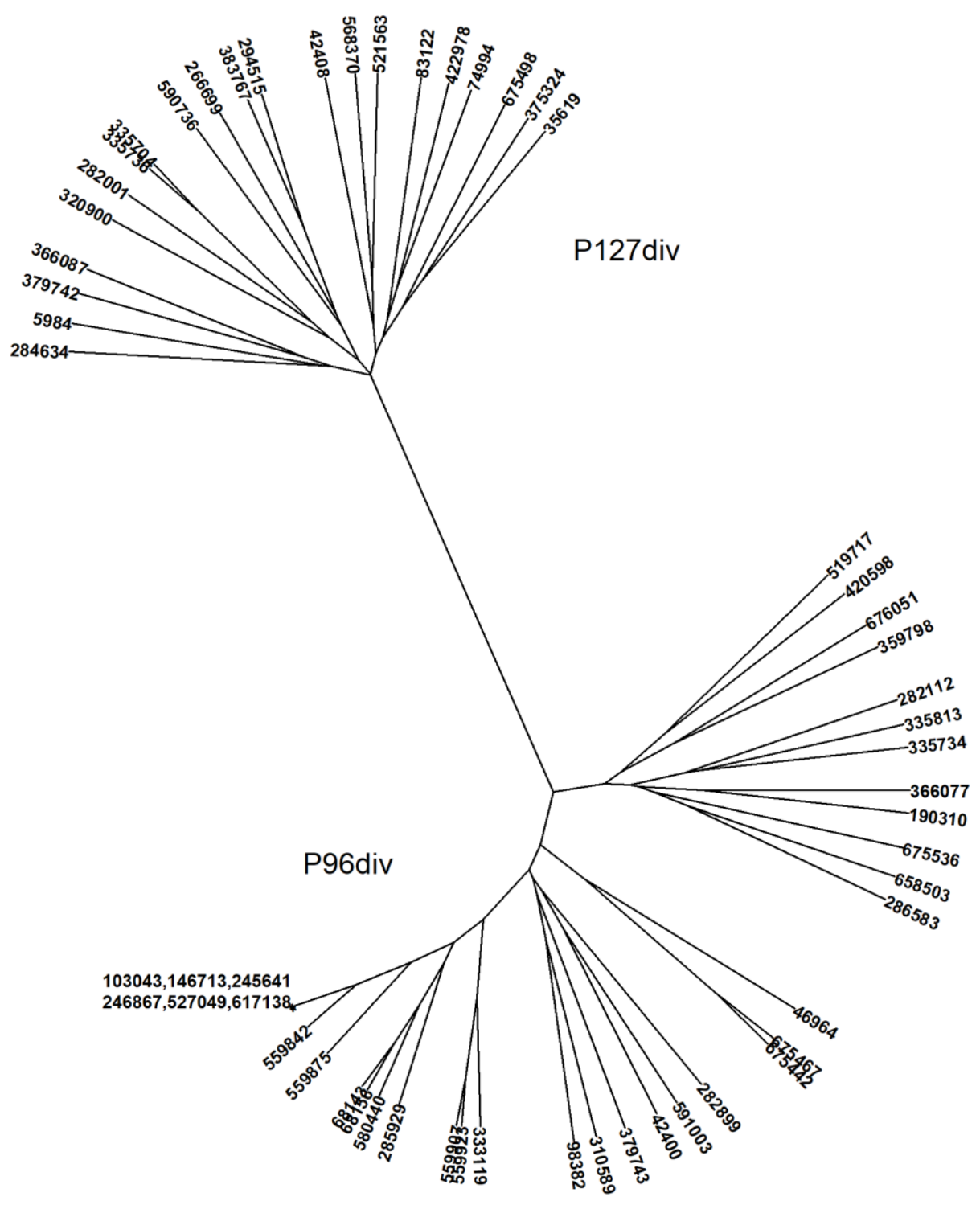

Fig. 4 Hierarchical clustering mtSNPs based on 1- $\phi$. Two sets of SNPs are shown with the diverse scores distinct between P127 and P96. More differentiations in SNP variation are observed in P96. *: $6 \mathrm{mtSNPs}$ with $\phi=1$ but not adjacent.

Table 2 Population diversity and annotation of mtSNPs. A summary of $57 \mathrm{mtSNPs}$ including same column information as described in Table 1 . Here, SNP id with prefix "mt" denoting mitochondrial origin. Gene rrn5 is located inside of rrn18 denoted as rrn18/rrn5. 


\begin{tabular}{|c|c|c|c|c|c|c|}
\hline id & $r e f$ & alt & $D P$ & P96div & P127div & annotation \\
\hline $\mathrm{mt5984}$ & $\mathrm{C}$ & $\mathrm{T}$ & 310 & 0.03 & 0.15 & Copia3-SB \\
\hline mt35619 & $\mathrm{G}$ & A & 163 & 0.15 & 0.03 & atp9 \\
\hline $\mathrm{mt} 42400$ & $\mathrm{C}$ & $\mathrm{T}$ & 189 & 0 & 0.18 & \\
\hline mt42408 & A & $\mathrm{G}$ & 189 & 0 & 0.18 & \\
\hline mt46964 & $\mathrm{G}$ & A & 911 & 0.09 & 0 & $\operatorname{nad} 4$ \\
\hline mt68143 & $\mathrm{T}$ & $\mathrm{C}$ & 27391 & 0.11 & 0 & \\
\hline mt68156 & $\mathrm{G}$ & A & 13853 & 0.15 & 0.01 & \\
\hline mt74994 & $\mathrm{C}$ & A & 325 & 0.01 & 0.19 & \\
\hline $\mathrm{mt} 83122$ & $\mathrm{~T}$ & $\mathrm{C}$ & 74 & 0 & 0.13 & rps1 \\
\hline mt98382 & G & A & 274 & 0.16 & 0 & \\
\hline mt103043 & A & $\mathrm{C}$ & 8620 & 0.29 & 0 & \\
\hline mt146713 & $\mathrm{C}$ & A & 27772 & 0.29 & 0 & \\
\hline mt190310 & $\mathrm{T}$ & $\mathrm{C}$ & 111 & 0.04 & 0.19 & \\
\hline $\mathrm{mt} 245641$ & G & A & 7111 & 0.29 & 0 & \\
\hline$m t 246867$ & $\mathrm{G}$ & $\mathrm{T}$ & 7127 & 0.29 & 0 & \\
\hline mt266699 & G & A & 44 & 0 & 0.12 & \\
\hline $\mathrm{mt} 282001$ & $\mathrm{~T}$ & A & 202 & 0 & 0.23 & \\
\hline $\mathrm{mt} 282112$ & $\mathrm{C}$ & $\mathrm{T}$ & 114 & 0.21 & 0.07 & \\
\hline mt282899 & $\mathrm{C}$ & A & 249 & 0.01 & 0.12 & \\
\hline $\mathrm{mt} 284634$ & $\mathrm{G}$ & $\mathrm{C}$ & 225 & 0.13 & 0.01 & \\
\hline$m t 285929$ & GAAAAAAAA & GAAAAAAAAA & 148 & 0.12 & 0 & \\
\hline $\mathrm{mt} 286583$ & $\mathrm{G}$ & $\mathrm{T}$ & 408 & 0.06 & 0.48 & \\
\hline $\mathrm{mt} 294515$ & $\mathrm{C}$ & $\mathrm{T}$ & 530 & 0.04 & 0.21 & \\
\hline mt310589 & $\mathrm{T}$ & A & 111 & 0.15 & 0.02 & \\
\hline mt320900 & TAAAAA & TAAAAAA & 52 & 0.01 & 0.12 & \\
\hline mt333119 & $\mathrm{G}$ & A & 11831 & 0.11 & 0 & atp4 \\
\hline mt335704 & G & A & 899 & 0.04 & 0.19 & atp4 \\
\hline mt335734 & $\mathrm{G}$ & $\mathrm{T}$ & 899 & 0.17 & 0 & atp4 \\
\hline $\mathrm{mt} 335736$ & $\mathrm{~T}$ & $\mathrm{C}$ & 899 & 0.07 & 0.26 & atp4 \\
\hline $\mathrm{mt} 335813$ & $\mathrm{G}$ & $\mathrm{C}$ & 525 & 0.17 & 0 & atp4 \\
\hline mt359798 & $\mathrm{C}$ & $\mathrm{T}$ & 93 & 0 & 0.11 & \\
\hline mt366077 & A & $\mathrm{T}$ & 202 & 0 & 0.08 & \\
\hline mt366087 & A & $\mathrm{G}$ & 202 & 0 & 0.08 & \\
\hline mt375324 & $\mathrm{T}$ & $\mathrm{G}$ & 1786 & 0.09 & 0 & Gypsy-53 \\
\hline mt379742 & G & A & 56 & 0 & 0.08 & \\
\hline mt379743 & $\mathrm{C}$ & $\mathrm{T}$ & 56 & 0.08 & 0 & \\
\hline mt383767 & $\mathrm{C}$ & $\mathrm{T}$ & 124 & 0.03 & 0.16 & \\
\hline mt420598 & G & A & 622 & 0.32 & 0.08 & \\
\hline $\mathrm{mt} 422978$ & $\mathrm{C}$ & $\mathrm{T}$ & 124 & 0 & 0.13 & \\
\hline mt519717 & G & A & 174 & 0.26 & 0.09 & $\mathrm{rrn} 18 / \mathrm{rrn} 5 / \mathrm{ccmFC}$ \\
\hline mt521563 & G & A & 289 & 0.21 & 0 & $\mathrm{rrn} 18 / \mathrm{rrn} 5 / \mathrm{ccmFC}$ \\
\hline mt527049 & A & $\mathrm{C}$ & 13632 & 0.29 & 0 & $\operatorname{rrn} 18 / \mathrm{rrn} 5$ \\
\hline mt559842 & A & $\mathrm{C}$ & 10607 & 0.18 & 0 & rrn18/rrn5 \\
\hline mt559875 & G & $\mathrm{T}$ & 10281 & 0.1 & 0 & rrn18/rrn5 \\
\hline mt559907 & $\mathrm{C}$ & G & 10416 & 0.08 & 0 & rrn18/rrn5 \\
\hline mt559923 & $\mathrm{C}$ & $\mathrm{T}$ & 10387 & 0.12 & 0 & rrn18/rrn5 \\
\hline mt568370 & $\mathrm{C}$ & $\mathrm{T}$ & 244 & 0.15 & 0.02 & rrn18/rrn5 \\
\hline $\mathrm{mt} 580440$ & G & $\mathrm{T}$ & 149 & 0.13 & 0 & \\
\hline mt590736 & G & $\mathrm{T}$ & 195 & 0 & 0.08 & \\
\hline mt591003 & G & $\mathrm{C}$ & 89 & 0 & 0.1 & \\
\hline mt617138 & $\mathrm{C}$ & A & 11827 & 0.29 & 0 & \\
\hline mt658503 & $\mathrm{C}$ & $\mathrm{T}$ & 119 & 0.2 & 0.05 & \\
\hline mt675442 & $\mathrm{G}$ & $\mathrm{C}$ & 597 & 0.12 & 0.01 & \\
\hline
\end{tabular}




\begin{tabular}{llllrl} 
mt675467 & $\mathrm{C}$ & $\mathrm{T}$ & 114 & 0.2 & 0.03 \\
$\mathrm{mt} 675498$ & $\mathrm{G}$ & $\mathrm{A}$ & 571 & 0.11 & 0.01 \\
$\mathrm{mt} 675536$ & $\mathrm{C}$ & $\mathrm{T}$ & 571 & 0.11 & 0.01 \\
$\mathrm{mt} 676051$ & $\mathrm{G}$ & $\mathrm{T}$ & 103 & 0.21 & 0.04 \\
\hline
\end{tabular}

Genetically, both 'P96' and 'P127' have undergone complex breeding crossing history. As is typical in breeding outcrossing perennial ryegrass, 'P96' was initiated as a cross between two breeding populations. This was achieved by crossing 18 pairs of individuals selected randomly from the respective populations. Seeds were harvested both parental populations and advanced through to $\mathrm{F}_{2}$ population, and after cycles of selection to form 'P96'. Therefore, 'P96' is cytoplasmic heterogeneous, with cytoplasm derived from the both breeding populations. While 'P127' was started as an inter-cross amongst five cultivar populations, but only seeds were harvested from one of parental cultivars and advanced to form 'P127'. So, the cytoplasm of 'P127' was only derived from a single cultivar. This largely explains the result that "P96" was more diverse than 'P127', revealed by mtSNPs (Fig. 1b). The overall diversity scores based on the mtSNPs (P96div $=0.15$, P127div $=0.11$, Fig. 4) indicated higher degree of diversity indeed occurred within 'P96' than that within 'P127'.

However, the population structures revealed by cpSNPs (Fig. 1a) remained unexplained with our current knowledge. Plastid (chloroplast or its precursor) inheritance is more intriguing. Among flowering plants, biparental inheritance has been reported more common in plastids than in mitochondria (Mogensen 1996), and chloroplast biparental inheritance was perceived as more common in outcrossing species (Reboud and Zeyl 1994).

\section{Cytoplasmic markers identified from a PstI-MspI GBS library}

GBS uses restriction enzymes for genome complexity reduction so there are many modifications in the choice of enzymes. The use of a two-enzyme PstI-MspI GBS protocol (Poland et al. 2012) further reduces genome complexity and increases read depth for more reliably calling of nuSNPs. It was therefore of interest to evaluate how this double-enzyme GBS protocol would affect cytoplasmic SNP marker discovery. To this end, a dataset based on another two ryegrass populations, 'S96' and 'S127', was recruited. From the two respective populations 45 and 49 plants were sampled. All the samples underwent the PstI-MspI digestion in one GBS library construction.

Out of $1,545,311$ tags 1,038 unique ones were mapped to ryegrass cpDNA and 4,631 to mtDNA. In total, 337 SNPs were identified via the same procedures as applied to the ApeKI libraries. Of these 337 SNPs 65.9\% SNPs (222) were uniformly distributed between 'S96' and 'S127', varied only with the corresponding reference sites. After removal of the uniform SNPs 115 SNPs (23 cpSNPs and 92 mtSNPs) were retained for statistical analysis. The similar results were obtained as that from the 
ApeKI data. MDS analysis based on cpSNPs (Fig. 5a) showed no overall difference between populations, but discernible within-population variation. And the variations due to mtSNPs largely explained the two populations (Fig. 5b).

\section{a: based on 23 cpSNPs}

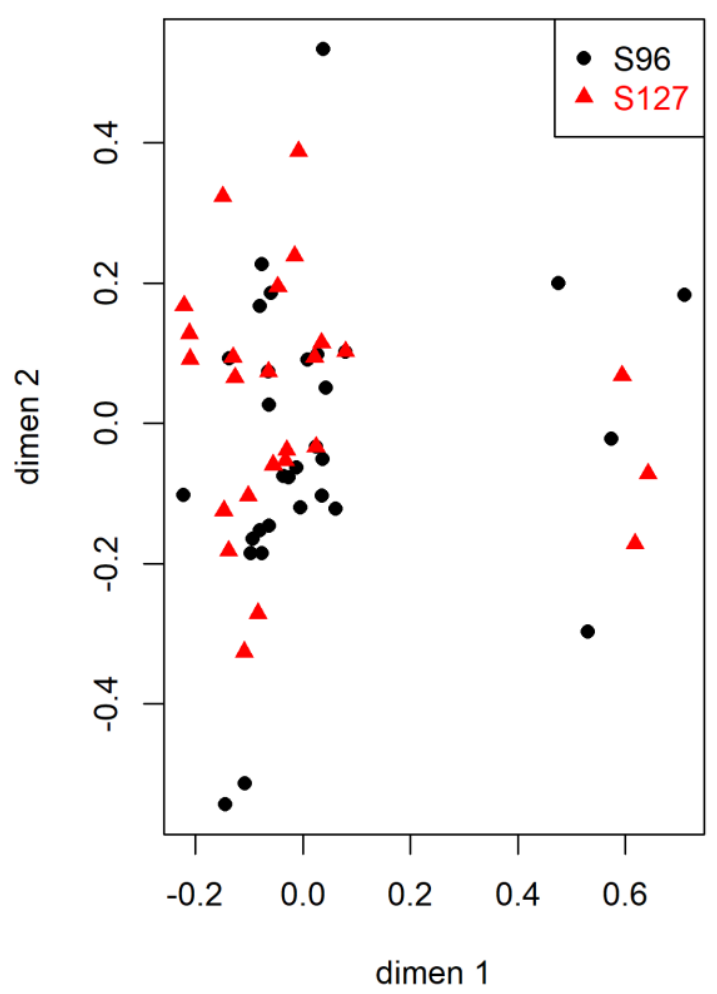

b: based on $92 \mathrm{mtSNPs}$

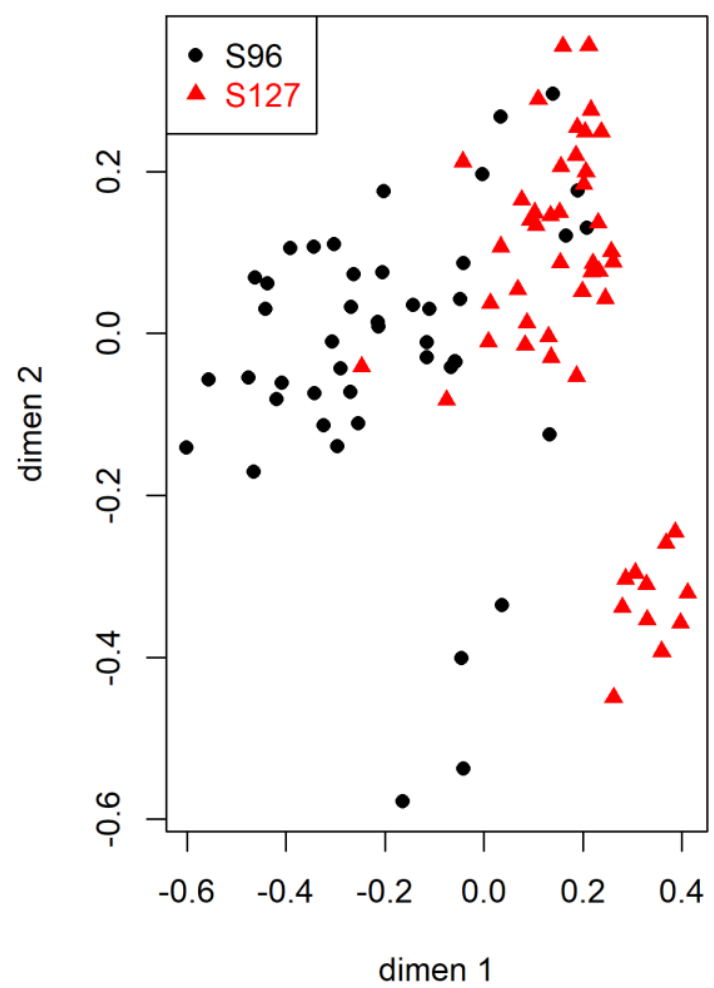

Fig. 5 Multidimensional scaling (MDS) clustering of samples based on cpSNPs (a) and mtSNPs (b), from a PstI-MspI library.

Based on the same criteria we used for ApeKI data (BH adjusted p-value < 0.01) only 12 SNPs showed significantly different between 'S96' and 'S127' (Table 3), among which adjacent SNPs (cp60150/60167, mt636996/636998, mt664895/664898/664924) were all perfectly correlated $(\phi=1)$.

Table 3 SNPs called from a PstI-MspI GBS library. A summary of SNPs including the same column information as described in Table 1 and 2.

\begin{tabular}{lllrrrr} 
id & ref & alt & DP & S96div & S127div & annotation \\
\hline cp54471 & G & A & 721 & 0.02 & 0.47 & rbcL \\
cp60150 & G & A & 54 & 0.2 & 0 & $\operatorname{petA}$ \\
cp60167 & C & G & 54 & 0.2 & 0 & petA \\
mt32942 & T & C & 256 & 0.27 & 0 & \\
mt74994 & C & A & 1547 & 0.44 & 0 & \\
mt197479 & GTT & GT & 125 & 0 & 0.49 & \\
mt465677 & CAA & CA & 122 & 0.49 & 0.16 &
\end{tabular}




\begin{tabular}{rllrll}
$\mathrm{mt} 636996$ & $\mathrm{C}$ & $\mathrm{G}$ & 269 & 0.49 & 0 \\
$\mathrm{mt} 636998$ & $\mathrm{G}$ & $\mathrm{A}$ & 269 & 0.49 & 0 \\
$\mathrm{mt} 664895$ & $\mathrm{G}$ & $\mathrm{A}$ & 68 & 0.22 & 0 \\
$\mathrm{mt} 664898$ & $\mathrm{C}$ & $\mathrm{T}$ & 68 & 0.22 & 0 \\
$\mathrm{mt} 664924$ & $\mathrm{C}$ & $\mathrm{A}$ & 68 & 0.22 & 0 \\
\hline
\end{tabular}

For the three significant cpSNPs (Table 3), cp54471 from gene rbcL remained homogeneous within 'S96' but was heterogeneous (S127div = 0.47) in 'S127', and cp60150/cp60167 from gene petA (cytochrome f) were diverse only in 'S96'. All the mtSNPs (Table 3) were derived from the unannotated regions. mt636996 and mt664895 were derived from a region where several Gypsy transposons are located (Supplementary data 1). We have observed the number of nuSNPs is much less from PstI-MspI library than that from ApeKI library (our unpublished data), and the same was observed here for the cytoplasmic markers.

The 95 samples were generated from one PstI-MspI GBS library and sequenced in two lanes on the same flowcell. This, therefore, confirmed that the sample clustering based on cp or mtSNPs was not confounded by the technical factors of library batch or flowcell.

We have also checked whether the sequencing tags of fungal endophyte origin could possibly affect the observed population structures, as endophytes (Epichloë spp.) are known to naturally reside in $L$. perenne cultivar populations. We checked this by mapping all the tags (PstI-MspI library), that have been mapped to ryegrass cytoplasmic genomes, to an endophyte reference genome (Epichloë festucae, NCBI Accession PRJNA51625). No single SNP was obtained after following the same SNP calling procedures. Therefore, the endophyte factor hypothesized to influence the observed ryegrass population substructures was ruled out.

\section{Discussion}

We have demonstrated the effective discovery of cytoplasmic genome-wide markers based on genotyping-by-sequencing data in perennial ryegrass. This methodology enables us to characterize large number of samples, to survey gene variant right across cytoplasmic genomes, and to monitor within-population variations of chloroplast or mitochondrial origin.

Our approach depends on the assumption that sequencing tags derived from cpDNA and mtDNA must be available along with that from nuDNA, and the number of tags should be sufficiently large for reliable SNP detection. Provided hundreds and thousands of chloroplasts and mitochondria exist in each plant cell (Cole 2016), that assumption has also been proved true in this study. We have examined and then removed several possible factors, including GBS libraries, sequencing batches and 
endophyte, which might affect the observed within-population structures. Nonetheless, we recognize, compared to that revealed by nuSNPs (Supplementary Fig. 3), the within-population variation is of a different pattern, and more subtle substructures occurred. Robust modelling of noise level (e.g. to determine the threshold of SNP diversity score) should enhance signals and improve the estimate of accurate biological variations within a population. On the other hand, for a certain ryegrass population, the cytoplasm of its maternal population may still be of a mixed origin (with seeds harvested from both parents in earlier generations), which may contribute the observed complex patterns. It lacks methods to characterize systematically cytoplasmic genetic changes over many generations. Our methods now pave a way to design experiments to monitor cytoplasmic genetic changes in response to different breeding crossing schema, and to quantify the strength of biparental inheritance of chloroplast in ryegrass and other plants.

Maternal inheritance of cytoplasmic genes is widely accepted, but the exact mode of action varies from species to species (Reboud and Zeyl 1994), and largely remains to be elucidated (Birky 2001; Luo et al. 2018). CMS (cytoplasmic male sterility) gene and its use in crop production have been widely-studied in plants (Chase 2007; McDermott et al. 2008), and served as a model for the study of cytoplasmic-nuclear interaction. However, the strength of cytoplasmic-nuclear interaction (Dobler et al. 2014) and co-evolution of cytonuclear integration (Sloan et al. 2018) need to be assessed accurately. Pertaining to plant improvement, sequence variation of cytoplasmic genomes has been found associated with adaptability (Galtier et al. 2009; Bock et al. 2014). Markers derived from mtDNA and cpDNA were found polymorphic among barley cultivars (Hisano et al. 2016), or among individual plants of the same cultivar (Diekmann et al. 2009; Diekmann et al. 2012), but with a few plants genotyped. Cytoplasmic-nuclear interactions are under-explored in plant breeding. Cytoplasmic genome-wide markers as we developed here will facilitate the further understanding of cytoplasmic genome-wide variation by conducting systematic investigations of cytonuclear interactions. This can be achieved without the limitation of the models based on a few genes and a few samples.

In conclusion, we have demonstrated that cytoplasmic markers are readily identified from existing genotyping-by-sequencing (GBS) workflow. The cytoplasmic genome-wide markers contribute a new dimension of genetic information for characterizing breeding populations. Analyses based on markers from chloroplasts and mitochondria reveal different population substructures, providing realizable opportunities to study its mode of action upon selections. Further improvement will be made in assessing sensitivity in marker discovery and accuracy in marker utilization. Our methods invite indepth exploration of the implications and applications of cytoplasmic inheritance in many other plant species.

\section{Materials and methods}


bioRxiv preprint doi: https://doi.org/10.1101/610543; this version posted April 16, 2019. The copyright holder for this preprint (which was not certified by peer review) is the author/funder, who has granted bioRxiv a license to display the preprint in perpetuity. It is made available under aCC-BY-NC-ND 4.0 International license.

\section{GBS data from perennial ryegrass populations}

Genotyping-by-sequencing (GBS) have been conducted in our lab for numerous perennial ryegrass populations including elite breeding lines and cultivars. GBS library construction, DNA sequencing and data generation were described in a previous publication (Faville et al. 2018). Raw sequencing read data from four ryegrass populations were selected in this study, with two (designated as 'P96' and 'P127') having sequence data from ApeKI GBS libraries, and two ('S96' and 'S127') from a PstIMspI library. Populations 'P96' and 'P127' consisted of 112 and 120 individuals, respectively, while 'S96' and 'S127' contained 45 and 49 individuals, respectively. GBS libraries comprising these individuals were sequenced twice and reads from the two sequencing lanes were combined.

\section{Tag sequence processing, alignment and SNP calling}

Sequencing was conducted on HiSeq2000 as 101 bp single-end reads. Quality reads (having a barcode and a cut site, and no N's) were processed and de-multiplexed using a Java module "GBSSeqToTagDPPlugin" from the TASSEL-GBS pipeline (Glaubitz et al. 2014). The quality reads (referred to as tags hereafter) of $64 \mathrm{nt}$ were saved in a SQLite database, from which we retrieved tag sequences and tag counts. "TagExportToFastqPlugin" was used to export tag sequences into a fastq file; tag counts in each individual sample were retrieved using "GetTagTaxaDistFromDBPlugin". There were redundant tags from each sample and we retained the redundant tags for calling SNPs in this report. We found similar population structures were resulted by using the unique set of tags for each sample.

The Lolium perenne chloroplast genome (135,282 bp, GenBank: AM777385.2) and mitochondrial genome (678,580 bp, GenBank: JX999996.1) and its associated annotations were downloaded and used as reference genomes. GBS tags were mapped to the reference genomes using "bwa-mem" (Li 2013). Functions from "samtools" ( $\mathrm{Li}$ et al. 2009) were used for alignment data conversion, indexing and sorting. SNP calling was conducted using "bcftools mpileup" and "bcftools call" (Li 2011) with ploidy $=1$ to call site genotype of $\{\mathrm{A}, \mathrm{T}, \mathrm{C}, \mathrm{G}\}$. Two genotype notations were used here and may be referred to as "letter genotype" and "number genotype". Letter genotype of one SNP like "G > A" represents "ref base $>$ alt base", where A is called at the position and different from the reference site $(G)$. And " $G>G$ " means that the site base $G$ is the same as the reference base G. For statistical analysis number genotype was used with, for example, " $G>G$ " is encoded as 0 , and " $G>A$ " as 1 .

\section{Statistical analysis}

Because of the binary nature of haploid cytoplasmic SNP data Phi correlation coefficient and Hamming distance were employed to calculate the genetic relationships between individual plants, 
bioRxiv preprint doi: https://doi.org/10.1101/610543; this version posted April 16, 2019. The copyright holder for this preprint (which was not certified by peer review) is the author/funder, who has granted bioRxiv a license to display the preprint in perpetuity. It is made available under aCC-BY-NC-ND 4.0 International license.

and to measure correlations between SNPs. Multivariate statistics was performed using R functions including "prcomp" for principal component analysis, and "cmdscale" for multi-dimensional scaling analysis. Alignment data interrogation and SNP data analysis were performed based on a number of Bioconductor packages (Lawrence et al. 2013), BioJulia packages (https://github.com/BioJulia) and visualized using IGV (Robinson et al. 2011).

\title{
Code availability
}

Scripts developed for SNP calling and data manipulation can be accessed from https://github.com/AgResearch/cpmt, under the license of GNU GPLv3.

\author{
Abbreviations \\ rp114 ribosomal protein L14 \\ psbA photosystem II protein D1 \\ psbB photosystem II CP47 chlorophyll apoprotein \\ petN cytochrome b6/f complex subunit $\mathrm{N}$ \\ atpA ATP synthase CF1 alpha subunit \\ psaA photosystem I P700 apoprotein A1 \\ psaB photosystem I P700 apoprotein A2 \\ ndhK NADH-plastoquinone oxidoreductase subunit K \\ atp9 ATP synthase subunits 9 \\ atp4 ATP synthase subunits 4 \\ nad4 NADH dehydrogenase subunit 4L \\ rps1 ribosomal protein $\mathrm{S} 14$ \\ rrn18 18S ribosomal RNA \\ rrn5 5S ribosomal RNA \\ ccmFC cytochrome $\mathrm{c}$ biogenesis \\ rbcL ribulose-1,5-bisphosphate carboxylase/oxygenase large subunit \\ petA cytochrome $\mathrm{f}$
}

\section{Author contributions}

MC conceived the scope of this study, developed the computing workflow and conducted data analysis. MC, MF, JJ and MC (Carena) conducted data interpretation and contributed to writing of the paper.

\section{Acknowledgements}

We thank Tom Lyons and Zulfi Jahufer for providing information on the breeding background of the ryegrass populations used in the study; Dan Sun and Alan McCulloch for maintaining HPC (highperformance computing) environment; and Brent Barrett for useful comments. We thank Grasslands Innovation Ltd, New Zealand for accessing to plant materials and genotyping-by-sequencing raw read data. 
bioRxiv preprint doi: https://doi.org/10.1101/610543; this version posted April 16, 2019. The copyright holder for this preprint (which was not certified by peer review) is the author/funder, who has granted bioRxiv a license to display the preprint in perpetuity. It is made available under aCC-BY-NC-ND 4.0 International license.

\section{Conflict of interest}

The authors declare that they have no conflict of interest.

\section{References}

Bao W, Kojima KK, Kohany O (2015) Repbase update, a database of repetitive elements in eukaryotic genomes. Mob DNA 6:11. doi: 10.1186/s13100-015-0041-9

Birky CW (2001) The inheritance of genes in mitochondria and chloroplasts: laws, mechanisms, and models. Annu Rev Genet 35:125-148. doi: 10.1146/annurev.genet.35.102401.090231

Bock DG, Andrew RL, Rieseberg LH (2014) On the adaptive value of cytoplasmic genomes in plants. Mol Ecol 23:4899-4911. doi: 10.1111/mec.12920

Chase CD (2007) Cytoplasmic male sterility: a window to the world of plant mitochondrial-nuclear interactions. Trends Genet 23:81-90. doi: 10.1016/j.tig.2006.12.004

Cole LW (2016) The evolution of per-cell organelle number. Front Cell Dev Biol 4: . doi: $10.3389 /$ fcell.2016.00085

Daniell H, Lin C-S, Yu M, Chang W-J (2016) Chloroplast genomes: diversity, evolution, and applications in genetic engineering. Genome Biol 17:134. doi: 10.1186/s13059-016-1004-2

Diekmann K, Hodkinson TR, Barth S (2012) New chloroplast microsatellite markers suitable for assessing genetic diversity of Lolium perenne and other related grass species. Ann Bot 110:1327-1339. doi: 10.1093/aob/mcs044

Diekmann K, Hodkinson TR, Wolfe KH, van den Bekerom R, Dix PJ, Barth S (2009) Complete chloroplast genome sequence of a major allogamous forage species, perennial ryegrass (Lolium perenne 1.). DNA Res 16:165-176. doi: 10.1093/dnares/dsp008

Dierckxsens N, Mardulyn P, Smits G (2017) NOVOPlasty: de novo assembly of organelle genomes from whole genome data. Nucleic Acids Res 45:e18. doi: 10.1093/nar/gkw955

Dobler R, Rogell B, Budar F, Dowling DK (2014) A meta-analysis of the strength and nature of cytoplasmic genetic effects. J Evol Biol 27:2021-2034. doi: 10.1111/jeb.12468

Elshire RJ, Glaubitz JC, Sun Q, Poland JA, Kawamoto K, Buckler ES, Mitchell SE (2011) A robust, simple genotyping-by-sequencing (GBS) approach for high diversity species. PLOS ONE 6:e19379. doi: 10.1371/journal.pone.0019379

Faville MJ, Ganesh S, Cao M, Jahufer MZZ, Bilton TP, Easton HS, Ryan DL, Trethewey JAK, Rolston MP, Griffiths AG, Moraga R, Flay C, Schmidt J, Tan R, Barrett BA (2018) Predictive ability of genomic selection models in a multi-population perennial ryegrass training set using genotyping-by-sequencing. Theor Appl Genet 131:703-720. doi: 10.1007/s00122-017-3030-1

Galtier N, Nabholz B, Glémin S, Hurst GDD (2009) Mitochondrial DNA as a marker of molecular diversity: a reappraisal. Mol Ecol 18:4541-4550. doi: 10.1111/j.1365-294X.2009.04380.x

Glaubitz JC, Casstevens TM, Lu F, Harriman J, Elshire RJ, Sun Q, Buckler ES (2014) TASSEL-GBS: A high capacity genotyping by sequencing analysis pipeline. PLOS ONE 9:e90346. doi: 10.1371/journal.pone.0090346 
bioRxiv preprint doi: https://doi.org/10.1101/610543; this version posted April 16, 2019. The copyright holder for this preprint (which was not certified by peer review) is the author/funder, who has granted bioRxiv a license to display the preprint in perpetuity. It is made available under aCC-BY-NC-ND 4.0 International license.

Hand ML, Spangenberg GC, Forster JW, Cogan NOI (2013) Plastome sequence determination and comparative analysis for members of the Lolium-Festuca grass species complex. G3 GenesGenomesGenetics 3:607-616. doi: 10.1534/g3.112.005264

Hiesel R, von Haeseler A, Brennicke A (1994) Plant mitochondrial nucleic acid sequences as a tool for phylogenetic analysis. Proc Natl Acad Sci 91:634-638. doi: 10.1073/pnas.91.2.634

Hisano H, Tsujimura M, Yoshida H, Terachi T, Sato K (2016) Mitochondrial genome sequences from wild and cultivated barley (Hordeum vulgare). BMC Genomics 17:824. doi: 10.1186/s12864016-3159-3

Islam MS, Studer B, Byrne SL, Farrell JD, Panitz F, Bendixen C, Møller IM, Asp T (2013) The genome and transcriptome of perennial ryegrass mitochondria. BMC Genomics 14:202. doi: 10.1186/1471-2164-14-202

Lawrence M, Huber W, Pagès H, Aboyoun P, Carlson M, Gentleman R, Morgan MT, Carey VJ (2013) Software for computing and annotating genomic ranges. PLOS Comput Biol 9:e1003118. doi: 10.1371/journal.pcbi.1003118

Li H (2013) Aligning sequence reads, clone sequences and assembly contigs with BWA-MEM. ArXiv13033997 Q-Bio

Li H (2011) A statistical framework for SNP calling, mutation discovery, association mapping and population genetical parameter estimation from sequencing data. Bioinformatics 27:29872993. doi: 10.1093/bioinformatics/btr509

Li H, Handsaker B, Wysoker A, Fennell T, Ruan J, Homer N, Marth G, Abecasis G, Durbin R, 1000 Genome Project Data Processing Subgroup (2009) The sequence alignment/map format and samtools. Bioinforma Oxf Engl 25:2078-2079. doi: 10.1093/bioinformatics/btp352

Luo S, Valencia CA, Zhang J, Lee N-C, Slone J, Gui B, Wang X, Li Z, Dell S, Brown J, Chen SM, Chien Y-H, Hwu W-L, Fan P-C, Wong L-J, Atwal PS, Huang T (2018) Biparental inheritance of mitochondrial DNA in humans. Proc Natl Acad Sci 115:13039-13044. doi: 10.1073/pnas.1810946115

McDermott P, Connolly V, Kavanagh TA (2008) The mitochondrial genome of a cytoplasmic male sterile line of perennial ryegrass (Lolium perenne L.) contains an integrated linear plasmidlike element. Theor Appl Genet 117:459-470. doi: 10.1007/s00122-008-0790-7

Mogensen HL (1996) The hows and whys of cytoplasmic inheritance in seed plants. Am J Bot 83:383-404. doi: $10.2307 / 2446172$

Poland JA, Brown PJ, Sorrells ME, Jannink J-L (2012) Development of high-density genetic maps for barley and wheat using a novel two-enzyme genotyping-by-sequencing approach. PLoS ONE 7: . doi: 10.1371/journal.pone.0032253

Reboud X, Zeyl C (1994) Organelle inheritance in plants. Heredity 72:132-140 . doi: 10.1038/hdy.1994.19

Robinson JT, Thorvaldsdóttir H, Winckler W, Guttman M, Lander ES, Getz G, Mesirov JP (2011) Integrative genomics viewer. In: Nat. Biotechnol. https://www.nature.com/articles/nbt.1754. Accessed 11 Sep 2018 
Sloan DB, Warren JM, Williams AM, Wu Z, Abdel-Ghany SE, Chicco AJ, Havird JC (2018) Cytonuclear integration and co-evolution. Nat Rev Genet 19:635. doi: 10.1038/s41576-0180035-9

Tarailo-Graovac M, Chen N (2009) Using RepeatMasker to identify repetitive elements in genomic sequences. Curr Protoc Bioinforma 25:4.10.1-4.10.14. doi: 10.1002/0471250953.bi0410s25

Wolfe KH, Li WH, Sharp PM (1987) Rates of nucleotide substitution vary greatly among plant mitochondrial, chloroplast, and nuclear DNAs. Proc Natl Acad Sci USA 84:9054-9058 